\title{
Photoelectromagnetic Investigations of Graphene
}

\author{
M. NOWAK*, B. Solecka And M. JesioneK \\ Solid State Physics Section, Institute of Physics - Center for Science and Education, \\ Silesian University of Technology, Z. Krasińskiego 8, 40-019 Katowice, Poland
}

\begin{abstract}
The photoelectromagnetic investigations of graphene has been performed using noncontact technique. The dependence of photoelectromagnetic response on magnetic field induction, illumination intensity for different photon energies, and frequency of illumination chopping is presented. We anticipate our paper to be a starting point for investigations of carrier diffusion length in this material. Such investigations should be essential for development of graphene electronic and optoelectronic devices.
\end{abstract}

DOI: 10.12693 /APhysPolA.126.1104

PACS: 72.80.Vp, 73.50.Gr, 73.50.Pz, 78.67.Wj

\section{Introduction}

Outstanding properties of graphene [1] are of great importance for fundamental research as well as for a wide range of applications, e.g. in optoelectronics. However, while knowledge on minority carrier lifetime $(\tau)$ in graphene is important for realization of graphene-based devices, its value is poorly known.

Despite the fact that the photoelectromagnetic (PEM) methods of determining $\tau$ are very promising [2-5], to the best of our knowledge, the PEM investigations of graphene have not been reported yet. The PEM signal decreases with the increase of the recombination rate, i.e. with the decrease of $\tau$. It is worth to mention that using PEM method, very small carrier lifetimes (e.g., $\tau=$ $10^{-11} \mathrm{~s}$ in GaAs [6]) can be measured because the PEM signal in some cases [3] is proportional to the diffusion length of carriers $\left(L=(D \tau)^{1 / 2}\right.$, where $D$ is the diffusion coefficient). Of course, PEM signal is stronger for higher mobilities $(\mu)$ of carriers [3]. Measurements of electron transport in graphene and its bilayers yielded $\mu$ higher than $3 \times 10^{5} \mathrm{~cm}^{2} /(\mathrm{V} \mathrm{s})$ at $300 \mathrm{~K}[7]$. In this paper, we present the results of PEM investigations of graphene for the first time.

\section{Experiment}

The investigated samples of graphene were supplied by Graphene Supermarket. Monolayer graphene films were grown by CVD processing on a cooper foil and transferred onto a Corning EAGLE XGTM AMLCD glass substrate. Thickness of the glass substrates was $0.7 \mathrm{~mm}$. Graphene coverage was about $90 \%$. The polycrystalline graphene films were continuous, with occasional holes and cracks. Unfortunately the data on carrier mobility in graphene transferred on glass substrate is not available. In [8] the CVD graphene (purchased in

${ }^{*}$ corresponding author; e-mail: Marian.Nowak@polsl.pl
Graphene Supermarket) was transferred from its Cu foil onto the patterned electrodes on hafnia based selfassembled nanodielectrics. In this case the mean carrier mobility was equal to $2280 \mathrm{~cm}^{2} /(\mathrm{V} \mathrm{s})$ with a standard deviation of $880 \mathrm{~cm}^{2} /(\mathrm{V} \mathrm{s})$ [8].

The PEM investigations were done using noncontact technique [9-15]. Samples were placed in a slit between neodymium magnets and a measuring coil. The steady magnetic field induction was equal to $B_{1}=0.35 \mathrm{~T}$. The apparatus was placed in vacuum. The graphene was illuminated through a $2 \mathrm{~mm}$ diameter hole in one of the magnets using temperature stabilized laser diodes: Sanyo DL7032-001 $(\lambda=830 \mathrm{~nm}, P=100 \mathrm{~mW})$ or Roithner Laser Technique RLT650 $(\lambda=650 \mathrm{~nm}, P=200 \mathrm{~mW})$. The laser diodes were biased with ITC510 (Thorlabs) laser diode controller. In our experiments, the laser diode bias was sinusoidally modulated around a dc bias chosen to ensure that the waveform of the light output signal is sinusoidal with negligible distortion.

Free electrons and holes photogenerated in graphene by a circular spot diffused in all directions in the sheet. Because magnetic field was applied perpendicularly to the graphene surface, diffusing carriers were deflected owing to the Lorentz force and so-called PEM current flowed (Fig. 1). Under amplitude-modulated illumination of a sample the PEM circulating current varied, and consequently the changing magnetic flux, caused by it, induced a measurable voltage in suitably placed pickup coils. This voltage was measured using an EG\&G lock-in 5110 amplifier whose internal generator controlled the frequency $(f)$ of laser beam chopping. The radiation intensity was changed using neutral density filters UV-NIR-FILTER-250-2000 nm (Quartzglas-Substrate, Oriel). It was monitored using a Hamamatsu S3399 photodiode whose short-circuit current was measured using another EG\&G 5110 lock-in amplifier. The time dependence of the illumination was determined using the Rigol Technologies DS1202CA oscilloscope, too. The temperature was measured using Lake Shore Cryotronics 211 temperature monitor. All measurements were performed at $294 \mathrm{~K}$. The experimental setup was computerized using IEEE-488 bus. 


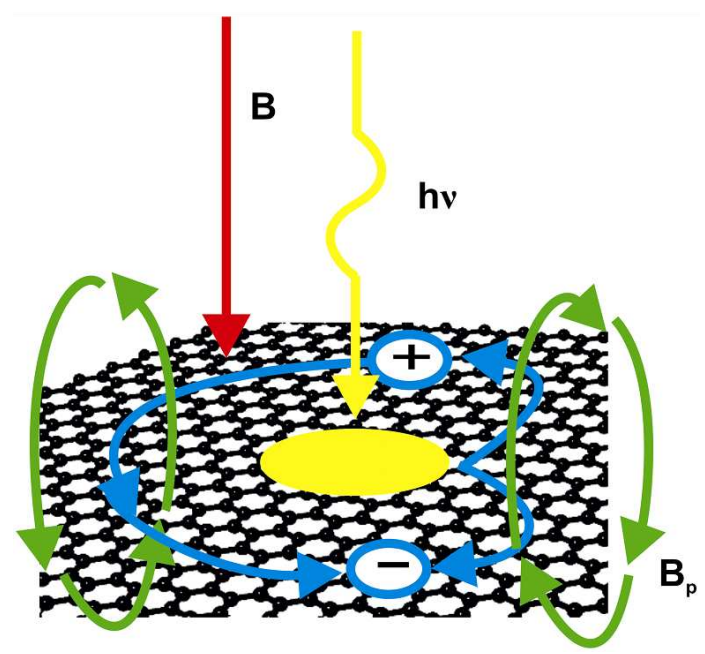

Fig. 1. Diagramic presentation of magnetic flux (broken curves $\boldsymbol{B}_{\boldsymbol{P}}$ ) evoked by the PEM current circulating in a point-illuminated graphene $(\boldsymbol{B}$ - the external magnetic field, $\oplus$ and $\ominus$ photogenerated holes and electrons, $\mathrm{h} \nu$ - photons, solid curves - trajectories of carriers).

\section{Results and discussion}

Figure 2 shows a typical set of time dependences of voltage responses $\left(V_{\mathrm{PEM}}\right)$ registered in PEM contactless investigations of unilluminated and illuminated graphene. The $V_{\mathrm{PEM}}$ is the total voltage minus the dark signal measured screening the sample for the same frequency of laser beam chopping. Figure 3 presents linear dependence of $V_{\mathrm{PEM}}$ on illumination intensity.

Figure 4 shows voltage responses in PEM contactless investigations of graphene as a function of frequency of illumination chopping. The results were least-squares fitted with linear dependence appropriate for sinusoidal modulation of illumination and for relatively small carrier lifetime (i.e. for $4 \pi^{2} f^{2} \tau^{2} \ll 1$ ).

It should be noted that in theoretical description of this effect one should take into account not only the usual, diffusive PEM effect evoked by the gradient of excess carrier concentration [10], but also the optically induced NernstEttingshausen effect, a component caused by the electron photothermomagnetoelectric effect evoked by the gradient of free carrier temperature due to strong interaction between photogenerated hot carriers and the equilibrium free carriers, and a component evoked by the existence of a small number of the photogenerated hot carriers which interact weakly with the equilibrium free carriers.

\section{Conclusions}

For the first time measurements of PEM effect in graphene are presented. They are done using noncontact and nondestructive technique. This method has the following advantages. The possible contaminations of a

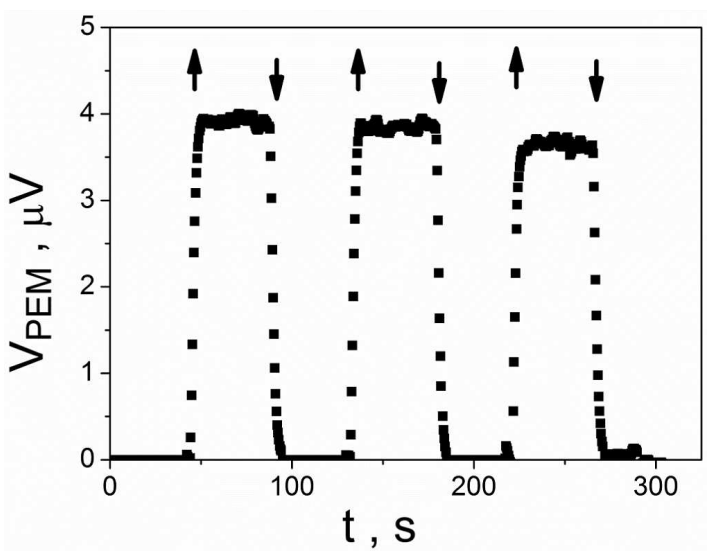

Fig. 2. Typical responses in PEM contactless investigations of graphene on the switch on $(\uparrow)$ and switch off $(\downarrow)$ of sinusoidally modulated illumination $\left(I_{0}=\right.$ $I_{\mathrm{MAX}}=2.6 \times 10^{22}$ photon $/\left(\mathrm{m}^{2} \mathrm{~s}\right), \lambda=830 \mathrm{~nm}, f=$ $72 \mathrm{kHz}, B=0.35 \mathrm{~T})$.

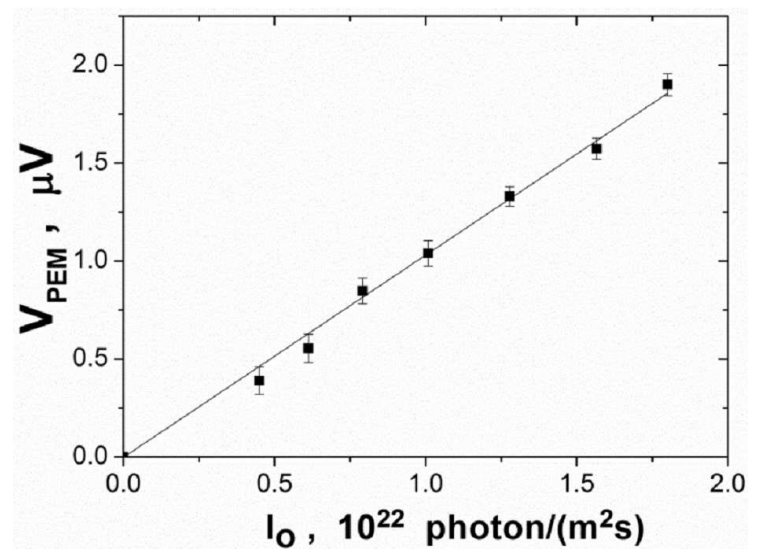

Fig. 3. Typical voltage responses in PEM contactless investigations of graphene vs. illumination intensity (symbols - the experimental data, solid lines - linear dependences calculated for the best fitted values, $\lambda=644 \mathrm{~nm}, P=0.1 \mathrm{~W}, f=72 \mathrm{kHz}, \mathrm{B}=0.35 \mathrm{~T})$.

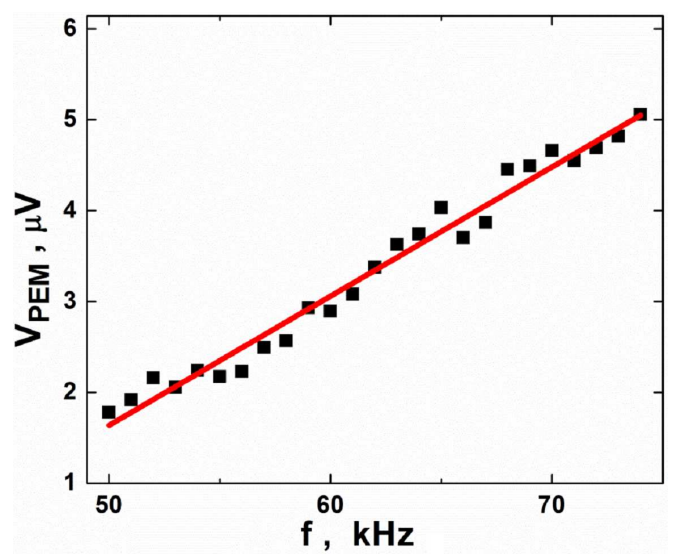

Fig. 4. Voltage responses in PEM contactless investigations of graphene vs. frequency of illumination chopping (symbols - the experimental data, solid line linear dependence calculated for the best fitted values of $A_{1}=1.42 \times 10^{-10} \mathrm{~V} \mathrm{~s}$ and $A_{0}=-5.47 \times 10^{-6} \mathrm{~V}$, $\lambda=830 \mathrm{~nm}, P=0.1 \mathrm{~W}, B=0.35 \mathrm{~T})$. 
sample during contact preparation, as well as the difficulties in the manufacturing of ohmic contacts are avoided. The distorting photovoltages evoked due to illumination of the nearcontact regions are eliminated. Studying the influence of gas adsorption, thermal annealing or etching on the recombination processes without the limitations caused by contacts is possible.

Future studies of the PEM effect in graphene should give the possibility to determine carrier diffusion length and the transport of energy in this attractive material. There are at least two possible methods of using PEM investigations for determining carrier diffusion length in graphene. The first one is based on the change of the profile of laser beam incident upon a sample. Profile of the beam affects radial distribution of photons in the illuminated spot and in consequence the gradient of the distribution of excess carriers. The latter gradient affects diffusion of the excess carriers from the illuminated to unilluminated part of a sample and influences the PEM current. However, the influence is different for different values of the carrier diffusion length. When one has theoretical description of the motion of carriers in the geometry of PEM measurements in graphene, the fitting of the experimental results registered for different laser beam profiles with theoretical formulae allows to determine the carrier diffusion length. The second method of determining carrier diffusion length in graphene is to use the appropriate theoretical description to fit the magnetic field dependence of the PEM results. Such a method is similar to the ones known for PEM investigations of bulk semiconductors. However, appropriate theoretical description of the PEM effect in graphene sample is needed.

Scanning a graphene sample with a light probe and performing the contactless PEM measurements, the recombination non-uniformity in the sample also can be measured. Such investigations should be essential for development of graphene electronic and optoelectronic devices. However, appropriate theoretical description of the PEM effect in graphene sample is needed that will take into account the photothermomagnetoelectric effects and the possible existence of hot carriers.

\section{Acknowledgments}

This work was partially supported by the National Science Centre project no. DEC-2012/05/B/ST7/01198.

\section{References}

[1] F. Bonaccorso, Z. Sun, T. Hasan, A.C. Ferrari, Nature Photon. 4, 611 (2010).

[2] M. Nowak, Prog. Quantum Electron. 11, 205 (1987).

[3] S. Kończak, M. Nowak, Phys. Status Solidi A 63, 305 (1981).

[4] M. Nowak, Phys. Status Solidi A 74, 313 (1982).

[5] M. Nowak, Phys. Status Solidi A 80, 691 (1983).

[6] K.D. Glinchuk, N.S. Zayatz, A.V. Prokhorovich, Fiz. Tekh. Poluprovodn. 16, 2214 (1982).

[7] S.V. Morozov, K.S. Novoselov, M.I. Katsnelson, F. Schedin, D.C. Elias, J.A. Jaszczak, A.K. Geim, Phys. Rev. Lett. 100, 016602 (2008).

[8] V.K. Sangwan, D. Jariwala, K. Everaerts, J.J. McMorrow, J. He, M. Grayson, L.J. Lauhon, T.J. Marks, M.C. Hersam, Appl. Phys. Lett. 104, 083503 (2014).

[9] J. Hlavka, Phys. Rev. B 6, 2325 (1972).

[10] J. Hlavka, Rev. Sci. Instrum. 52, 60 (1981).

[11] J. Hlavka, Rev. Sci. Instrum. 54, 1386 (1983).

[12] B. Loncierz, R. Murri, M. Nowak, Thin Solid Films 266, 274 (1995).

[13] B. Loncierz, M. Nowak, Proc. SPIE 3179, 151 (1997).

[14] M. Nowak, B. Solecka, Vacuum 57, 237 (2000).

[15] P. Duka, B. Solecka, M. Nowak, Proc. SPIE 5229, 329 (2003). 